\title{
Theoretical Study on the Dynamic Behavior of Pipes Conveying Gas-Liquid Flow
}

\author{
L. Enrique Ortiz-Vidal ${ }^{1, *}$, David G. Castillo ${ }^{2}$, and Quino Valverde ${ }^{2}$ \\ ${ }^{1}$ Mechanical Engineering Department, Sao Carlos School of Engineering, University of Sao Paulo, 13563-120, São Carlos/SP, Brazil \\ ${ }^{2}$ Engineering Department, Mechanical Engineering Section, Pontifical Catholic University of Peru, Av. Universitaria 1801, Lima, Peru
}

\begin{abstract}
The dynamic behavior of clamped-clamped straight pipes conveying gas-liquid two-phase flow is theoretically investigated, specifically the effect of the flow parameters on the frequency of the system. First, the equation of motion is derived based on the classic Païdoussis formulation. Assuming EulerBernoulli beam theory, small-deflection approximation and no-slip homogeneous model, a coupled fluid-structure fourth-order partial differential equation (PDE) is obtained. Then, the equation of motion is rendered dimensionless and discretized through Galerkin's method. That method transforms the PDE into a set of Ordinary Differential Equations (ODEs). The system frequency is obtained by solving the system of ODEs by allowing the determinant to be equal to zero. System frequencies for different geometries, structural properties and flow conditions have been calculated. The results show that the system frequency decreases with increasing two-phase flow velocity. By contrast, the former increases with increasing homogeneous void fraction. These theoretical results are in agreement with experimental findings reported in the literature. Furthermore, even for typical two phase flow conditions, the system can become unstable for inadequate chooses of geometry or material of the pipe.
\end{abstract}

\section{Introduction}

Pipes conveying gas-liquid two-phase flow are common in nuclear, process and oil\&gas industries, where the flow generates dynamics forces. Depending on the system conditions these forces may induce moderate or excessive structural vibrations, leading to structural instability. Then, a proper knowledge of the dynamic behavior of this kind of systems is relevant and has motivated relevant researches over the last five decades, see e.g. [1-4]. Fundamental experimental studies have shown a strong influence of two-phase flow parameters, such as mixture velocity, void fraction, flow pattern and slip, on the structural vibration response [5-9]. In the specific case of the frequency of the system, recently experimental results [8] indicate this parameter increases with increasing void fraction due to the effect of hydrodynamic mass (added mass). On the other hand, system frequency decreases with increasing two-phase mixture flow velocity. Theoretical studies on system frequency in order to imitate the effect of those two-phase flow parameters are needed. In general, the reported studies focused on determining the critical mixture velocity for reaching instability. In this paper, the dynamic stability of clamped-clamped straight pipes conveying gas-liquid two-phase flow is theoretically investigated, specifically the system frequency for typical two-phase flow conditions.

\section{Theory}

\subsection{Equation of the motion}

The mathematical formulation for pipes conveying fluid presented here is based on the classic Païdoussis formulation [10]. The system is composed by (i) a uniform pipe of length $L_{\text {span }}$, inner cross-sectional area $A$, internal perimeter $S$, linear mass density $m$, and flexural rigidity $E I$ and (ii) a two-phase fluid flow of linear mass density $M$ with mixture flow velocity $J$. The equation of motion is derived applying Newton's second law over free-body diagrams of the both fluid and pipe differential elements of Fig. 1. Euler-Bernoulli beam theory and small-deflection approximation are adopted. For the fluid element, in the $\mathrm{X}$ - and $\mathrm{Z}$ - directions, the following equations are obtained, respectively,

$$
\begin{aligned}
& -A \frac{\partial p}{\partial x}+q S-F \frac{\partial w}{\partial x}=M \frac{\partial J}{\partial t} \\
& A \frac{\partial}{\partial x}\left(p \frac{\partial w}{\partial x}\right)-q S \frac{\partial w}{\partial x}-F+M g \\
& =M\left[\frac{\partial^{2} w}{\partial t^{2}}+J^{2} \frac{\partial^{2} w}{\partial x^{2}}+2 J \frac{\partial^{2} w}{\partial x \partial t}+\frac{\partial J}{\partial t} \frac{\partial w}{\partial x}\right]
\end{aligned}
$$

where $w$ is the deflection of the pipe in the Z-direction. $p$ and $g$ are pressure and gravity, respectively. $q S$ and $F$ are related to reaction forces of the pipe on the two-phase fluid, in tangential and normal directions, respectively. 


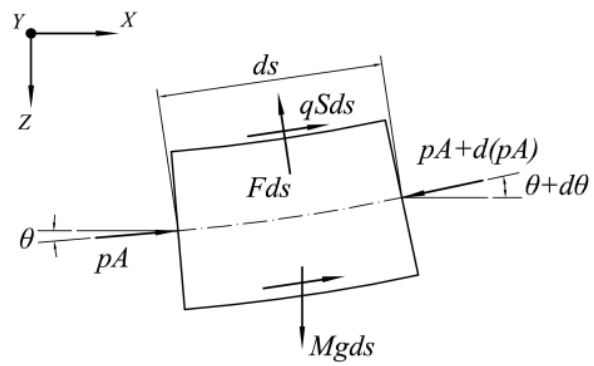

a)

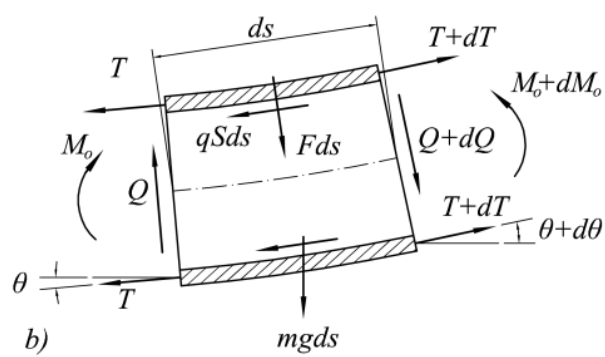

Fig. 1. Free-body diagrams of a differential element $d s$ of a horizontal pipe conveying fluid, (a) fluid and (b) pipe.

In the same form, for the pipe element, it is obtained,

$\frac{\partial T}{\partial x}-q S+F \frac{\partial w}{\partial x}=0$

$-\frac{\partial}{\partial x}\left(T \frac{\partial w}{\partial x}\right)+q S \frac{\partial w}{\partial x}+F+\frac{\partial Q}{\partial x}+m g=m \frac{\partial^{2} w}{\partial t^{2}}$

where $T$ and $Q$ are longitudinal tension and transverse shear force, respectively. Combining Eqs. (1)-(4), and using Païdoussis' procedure for calculation of $T$ and $Q$, gives the equation of the motion,

$E I \frac{\partial^{4} w}{\partial x^{4}}+(M+m) g\left((x-L) \frac{\partial^{2} w}{\partial x^{2}}+\frac{\partial w}{\partial x}\right)$

$+M J^{2} \frac{\partial^{2} w}{\partial x^{2}}+2 M J \frac{\partial^{2} w}{\partial t \partial x}+(M+m) \frac{\partial^{2} w}{\partial t^{2}}$

$+E I \frac{\mu}{\Omega} \frac{\partial}{\partial t}\left(\frac{\partial^{4} w}{\partial x^{4}}\right)=0$

where the terms represent respectively (from left to rigth): flexural force, pressure and tension, centrifugal force, Coriolis force, inertia force and structural damping force. $\Omega$ is the system frequency.

\subsection{Two-phase flow}

For the case of two-phase flow, the mixture flow velocity $J$, and the two-phase fluid mass $M$, can be represented by

$J=J_{G}+J_{L}$

$M=\rho_{M} A$

where $J_{G}$ and $J_{L}$ represent the superficial velocities of the gas and liquid, respectively. Superficial velocity of the phase is defined as the volumetric flow rate divided by inner cross-sectional area of the pipe. $\rho_{M}$ is the mixture density. This parameter depends on the mass density and volume fraction of each phase in the flow. Assuming no-slip homogeneous model [11], the mixture density can be calculated from homogeneous void fraction $(\lambda)$ by,

$\rho_{M}=\rho_{L}(1-\lambda)+\rho_{G} \lambda$

$\lambda=\frac{J_{G}}{J}$

For the purposes of this study, a two-phase flow condition is totally defined when both the mixture flow velocity $J$ and the homogeneous void fraction $\lambda$ are established.

\subsection{Nondimensional equation of motion}

The equation of the motion may be rendered dimensionless introducing the following parameters,

$\xi=\frac{x}{L}, \quad \eta=\frac{w}{L}, \quad \tau=\left(\frac{E I}{M+m}\right)^{1 / 2} \frac{t}{L^{2}}$

Thus, Eq. (5) becomes

$$
\eta^{\prime \prime \prime \prime}+\eta^{\prime \prime} j^{2}+2 \beta^{1 / 2} j \dot{\eta}^{\prime}+\ddot{\eta}=0
$$

where $\left({ }^{\circ}\right)$ and ()$^{\prime}$ indicates time $(\tau)$ and space $(\xi)$ derivate of parameter $\eta$. Eq. (11) represents the dimensionless equation of motion with neglected gravity and structural damping. The following expressions relate both dimensional and nondimensional parameters,

$$
\begin{aligned}
& j=\left(\frac{M}{E I}\right)^{1 / 2} J L_{\text {span }}, \quad \beta=\frac{M}{M+m}, \\
& \omega=\left(\frac{M+m}{E I}\right)^{1 / 2} \Omega L_{\text {span }}^{2}
\end{aligned}
$$

where $j$ and $\beta$ represent dimensionless velocity and mass ratio, respectively. $\omega$ is the dimensionless frequency of the system.

\subsection{Solution method}

Galerkin's method [10] is used to solve Eq. (11). This method transforms the distributed system into discrete one. Thus, the partial differential equation of the system becomes a set of ordinary differential equations. According to the method, the solution may be written as,

$$
\eta(\xi, \tau)=\sum_{r=1}^{N} \phi_{r}(\xi) q_{r}(\tau), \quad r=1,2, \ldots, N
$$

where the summation considers the first $r=N$ modes. $\phi_{r}(\xi)$ are the dimensionless eigenfunctions of a beam with clamped-clamped boundary conditions. $q_{r}(\tau)$ represent the generalized coordinates of the discretized system. Substituting Eq. (13) in Eq. (11), multiplying by $\phi_{s}(\xi)$ and integrating over the domain $[0,1]$, is obtained, 


$$
\sum_{r=1}^{N}\left\{\begin{array}{l}
\delta_{s r} \ddot{q}_{r}+\left[2 \beta^{1 / 2} j b_{s r}\right] \dot{q}_{r} \\
+\left[\delta_{s r} \lambda_{r}^{4}+j^{2} c_{s r}\right] q_{r}
\end{array}\right\}=0
$$

where

$\delta_{s r}=\int_{0}^{1} \phi_{s} \phi_{r} d \xi$

$b_{s r}=\int_{0}^{1} \phi_{s} \phi_{r}^{\prime} d \xi$,

$c_{s r}=\int_{0}^{1} \phi_{s} \phi_{r}^{\prime \prime} d \xi$

The parameter $\delta_{s r}$ represents the Kronecker delta. This is equal to 0 for $r \neq s$ and equal to 1 for $r=s$. For a clamped-clamped pipe, the constants $b_{s r}$ y $c_{s r}$ can be expressed by [12],

$$
\begin{aligned}
& b_{s r}= \begin{cases}\frac{4 \lambda_{r}^{2} \lambda_{s}^{2}}{\lambda_{r}^{4}-\lambda_{s}^{4}}\left\{(-1)^{r+s}-1\right\}, & s \neq r \\
0, & s=r\end{cases} \\
& c_{s r}=\left\{\begin{array}{lr}
\frac{4 \lambda_{r}^{2} \lambda_{s}^{2}}{\lambda_{r}^{4}-\lambda_{s}^{4}}\left(\lambda_{r} \sigma_{r}-\lambda_{s} \sigma_{s}\right)\left\{(-1)^{r+s}+1\right\}, s \neq r \\
\lambda_{r} \sigma_{r}\left(2-\lambda_{r} \sigma_{r}\right), & s=r
\end{array}\right.
\end{aligned}
$$

where $\lambda_{r}, \lambda_{s}, \sigma_{r}$ and $\sigma_{s}$ are parameters of the eigenproblem of a beam [13]. Eq. (14) can be expressed in standard form by,

$$
[\mathbf{M}] \ddot{q}+[\mathbf{C}] \dot{q}+[\mathbf{K}] q=0
$$

where $\mathbf{M}, \mathbf{C}$ and $\mathbf{K}$ are, respectively, mass, damping and stiffness matrices. In this paper, the system of Eq. (19) is solved adopting oscillatory solutions, i.e. $q=q_{r} e^{\mathrm{i} \omega \tau}$. Dimensionless frequencies of the system $\omega$, or eigenvalues, are found by allowing the determinant to be equal to zero for each specific flow condition.

\section{Results and Discussion}

Theoretical results for the dimensionless frequency of pipes conveying gas-liquid flow are presented. The two pipe systems of Table 1 are tested. CASE 1 and CASE 2 correspond to air-water two-phase flow in $3 / 4$ " and 1-1/2" commercial PVC pipes, respectively. In the case of the span length $L_{\text {span }}$, two lengths $\mathrm{L} 1=1.5 \mathrm{~m}$ and $\mathrm{L} 2=2.5 \mathrm{~m}$ are used.

\subsection{Verification of the formulation}

The CASE 1 pipe system with $\lambda=0$ is used to verify the presented formulation. This is equivalent to a pipe conveying liquid single-phase flow with $\beta=0.5$ (substituting the values of Table 1 on Eq. (12)). The results of dimensionless frequency of the system as a function of dimensionless velocity for the first three modes are shown in Fig 2. It can be observed that the Argand diagram of the complex frequencies is identical to the diagram presented in Païdoussis's book [10]. Some important matters should be pointed. First, the
Table 1. Tested pipe-systems conveying fluid.

\begin{tabular}{|l|r|r|}
\cline { 2 - 3 } \multicolumn{1}{c|}{} & \multicolumn{1}{c|}{ CASE 1 } & \multicolumn{1}{c|}{ CASE 2 } \\
\hline Outer diameter $(\mathrm{m})$ & 0.02667 & 0.04826 \\
Inner diameter $(\mathrm{m})$ & 0.02071 & 0.04078 \\
Pipe mass $(\mathrm{kg} / \mathrm{m})$ & 0.3362 & 0.7989 \\
Liquid density $\left(\mathrm{kg} / \mathrm{m}^{3}\right)$ & 998.2 & 998.2 \\
Gas density $\left(\mathrm{kg} / \mathrm{m}^{3}\right)$ & 1.49 & 1.49 \\
EI $(\mathrm{N} \cdot \mathrm{m} 2)$ & 37.93 & 313.3 \\
\hline
\end{tabular}

instability of the system occurs for the first mode, when, for the lowest dimensionless velocity, the imaginary part of frequency is different to zero. Then, the dimensionless frequency for $j=0$ (no-flow) is 22.3733. Finally, the dimensionless critical velocity (where instability occurs) is 6.28; approximately $2 \pi$ as pointed by [10].

\subsection{Frequency for two-phase flow}

Dimensionless frequency for two-phase flow has been calculated using the flow conditions of Fig. 3. We use the parameter Stability Level (Eq. (20)) to illustrate the results,

Stability Level $=\left(1-\frac{\omega_{r e f}-\omega_{i}}{\omega_{r e f}}\right) \cdot 100 \%$

where $\omega_{i}$ is the calculated dimensionless frequency of a specific point of Fig. 3. $\omega_{\text {ref }}$ represents the dimensionless frequency for no-flow liquid-filled pipe system and is equal to 22.3733. A diminution in the Stability Level value is associated to the reduction of dimensionless frequency of the system, and vice-versa. Moreover, Stability Level equals to 0 indicates that the system became unstable.

Fig. 4 shows Stability Level results as a function of two-phase flow parameters, for the CASE 1-L1 pipe system. Each one of the 42-points corresponds to the flow conditions of Fig. 3. For constant values of homogeneous void fraction, it is observed that Stability Level decreases with increasing the superficial velocity.

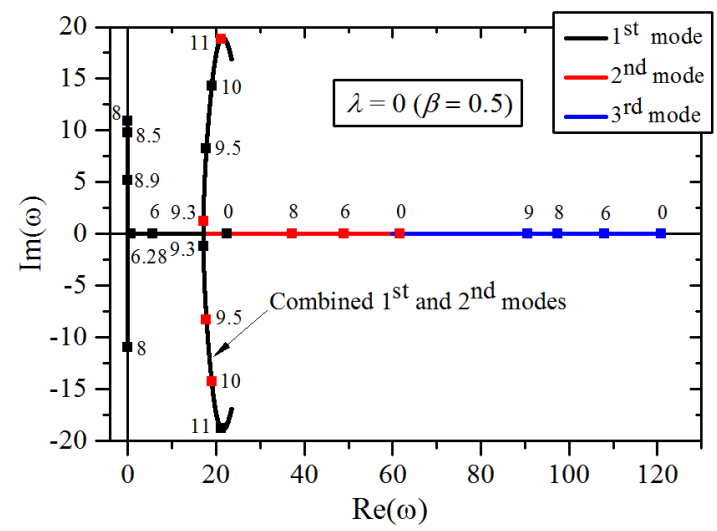

Fig. 2. Argand diagram of the complex frequencies of CASE 1 pipe system, considering $\lambda=0 \quad(\beta=0.5)$. Numbers indicate the values of dimensionless velocity $j$. 


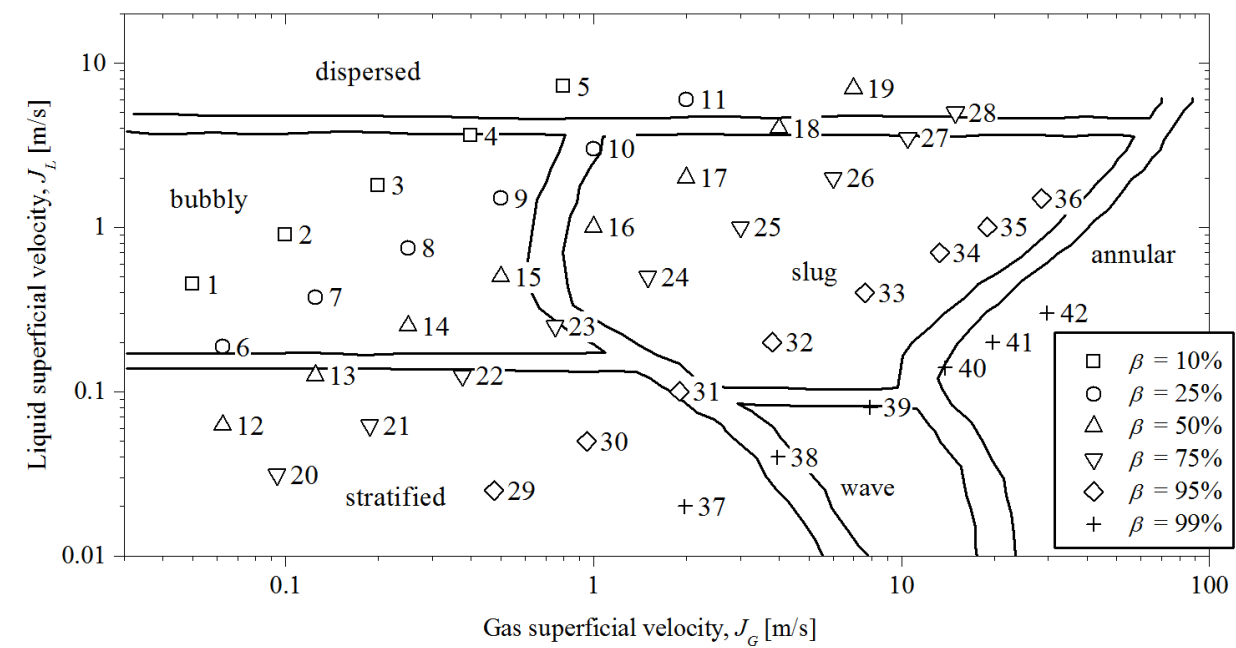

Fig. 3. Typical gas-liquid two-phase flow conditions on Mandhane et al. flow map [14]. The symbols are classified by homogeneous void fraction $\beta$.

For constant values of superficial velocity, an opposite behaviour of Stability Level is observed, i.e. Stability Level increases with increasing of homogeneous void fraction. This behaviour is due to the effect of hydrodynamic mass (added mass). Some values of Stability Level are presented in parenthesis. In general, the effect of velocity on the Stability Level is stronger than the effect of the homogeneous void fraction. These results are in agreement with experimental results reported by [8].

Fig 5 shows the Stability Level due to two-phase flow for three pipe systems. The effect of the pipe length $L_{\text {span }}$ is observed when compared results for CASE1-L1 and CASE1-L2. On the other hand, the effect of both geometry and mechanical properties of the pipe are taken into account when compared results for CASE 1-L2 and CASE 2-L2. It can be observed that an increment on the pipe length reduces the Stability Level of the system, making it unstable for two conditions. This fact is due to the pipe system becoming more slender. The opposite happens with the rigidity $E I$; for the same pipe length, i.e. CASE 1-L2 versus CASE 2-L2, the higher rigidity value, the larger Stability Level. The strong effect of the velocity on Stability Level is also evident in Fig. 5.

\section{Conclusions and Future Works}

The classical equation of pipes conveying fluid, incorporating the no-slip homogeneous two-phase model, captures properly the trend between two-phase flow parameters and the Stability Level of the system. The lower Stability Level, the smaller frequency of the system. Furthermore, frequency equals to 0 represents an unstable system. In agree with reported experimental results in the literature, theoretical simulations show that Stability level decreases with increasing mixture velocity; and, by contrast, it increases with increasing homogeneous void fraction. Also, inadequate combinations of two-phase flow conditions, geometry and mechanical properties of the pipe can lead to unstable systems.

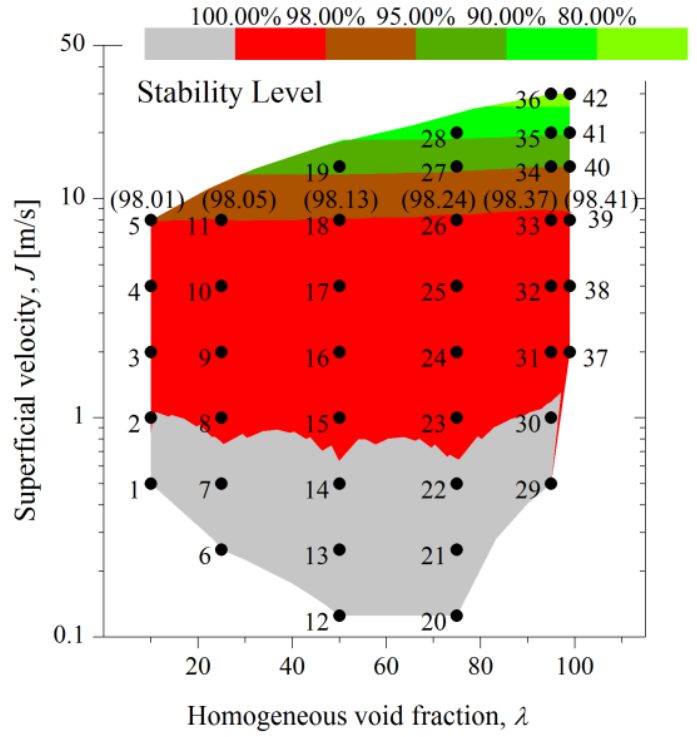

Fig. 4. Stability Level for the CASE 1-L1 pipe system. The numbers represent flow conditions of Fig. 3. The values in parenthesis indicate the Stability Level for two-phase flow conditions with $J=8 \mathrm{~m} / \mathrm{s}$.

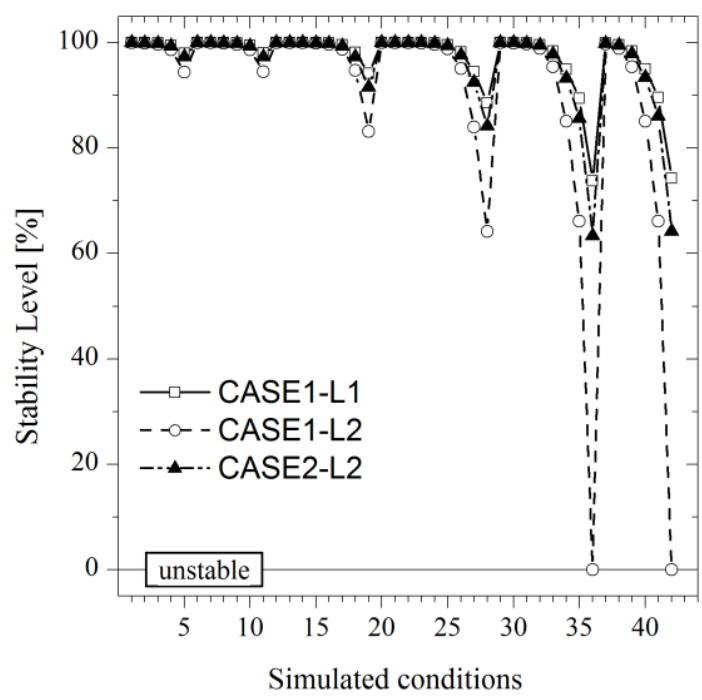

Fig. 5. Stability Level versus two-phase conditions of Fig. 3 for three pipe systems. 
Future works should perform comparisons with experimental results and analyse the influence of slip between phases, gravity and structural damping on the Stability level.

This research has been supported by CIENCIACTIVA del CONCYTEC. L. Enrique Ortiz Vidal is grateful to FIPAI.

\section{References}

1. K. Fujita, J. Wind Eng. Ind. Aerodyn. 33, 405 (1990).

2. M. J. Pettigrew, C. E. Taylor, N. J. Fisher, M. Yetisir, and B. A. W. Smith, Nucl. Eng. Des. 185, 249 (1998).

3. C. Monette and M. J. Pettigrew, J. Fluids Struct. 19, 943 (2004).

4. H. Chung, in Offshore Mech. Arct. Eng. Symp. (Dallas, TX, USA, 1985), pp. 398-402.

5. M. J. Pettigrew and C. E. Taylor, J. Press. Vessel Technol. Trans. ASME 116, 233 (1994).

6. C. Zhang, M. J. Pettigrew, and N. W. Mureithi, J. Press. Vessel Technol. 130, 11301 (2008).

7. T. Hibiki and M. Ishii, Nucl. Eng. Des. 185, 113 (1998).

8. L. E. Ortiz-Vidal, N. W. Mureithi, and O. M. H. Rodriguez, Nucl. Eng. Des. 313, 214 (2017).

9. Y. Geng, F. Ren, and C. Hua, Flow Meas. Instrum. 27, 113 (2012).

10. M. P. Païdoussis, Fluid-Structure Interactions: Slender Structures and Axial Flow, Vol. 1 (Elsevier Academic Press, 1998).

11. G. Wallis, One Dimensional Two-Phase Flow (McGraw-Hill, New York, 1969).

12. R. E. D. Bishop and D. C. Johnson, The Mechanics of Vibration (Cambridge University Press, 1979).

13. M. P. Paidoussis, Fluid-Structure Interacions: Slender Structures and Axial Flow (1998).

14. J. M. Mandhane, G. A. Gregory, and K. Aziz, Int. J. Multiph. Flow 1, 537 (1974). 